\title{
Analysis of Intrapulmonary Vessels and Epithelial-Endothelial Interactions in the Human Developing Lung
}

\author{
Sumiko Maeda, Satoshi Suzuki, Takashi Suzuki, Mareyuki Endo, Takuya Moriya, \\ Masayuki Chida, Takashi Kondo, and Hironobu Sasano \\ Department of Thoracic Surgery (SM, SS, MC, TK), Institute of Development, Aging and Cancer, Tohoku University, \\ and Department of Pathology (SM, TS, ME, TM, HS), Tohoku University School of Medicine, Sendai, Japan
}

\begin{abstract}
SUMMARY: The establishment of a sufficiently wide and functional blood-gas interface is of critical importance in lung development, but development of the intrapulmonary vascular system including alveolar capillary vessels still remains unclear. In this study, we first characterized the structural development of the vascular system in accordance with that of airways in human fetal lungs at the pseudoglandular phase $(8,13$, and 16 weeks gestation) by examining the immunohistochemical distribution of CD34 and $\alpha$-smooth muscle actin (SMA). Using double immunohistochemistry and 3-dimensional reconstruction techniques, endothelial cells in the developing lung could be classified into two different types according to the characteristics of their adjacent cells (presence or absence of SMA-positive cells) and their distribution (proximal or distal lung parenchyme). Endothelial cells without SMA-positive cells developed into a capillary network surrounding the budding components of distal airways during the mid-pseudoglandular phase before communicating with proximal vessels. We then examined the immunoreactivity of thrombomodulin and von Willebrand factor (VWF) in endothelial cells. Endothelial cells of the capillary network were mainly positive for VWF during the early gestational stages, but altered their phenotypes to those of mature lungs (vWF negative and thrombomodulin positive) during the terminal sac phase. We subsequently determined the immunohistochemical distribution of vascular endothelial growth factor (VEGF). Epithelial cells of the most distal airways were intensely positive for VEGF. These results suggest that VEGF present in airway epithelial cells is involved in the maturation as well as proliferation of capillary endothelial cells. Epithelial-endothelial interactions during lung development are considered very important in the establishment of the functional blood-gas interface. (Lab Invest 2002, 82:293-301).
\end{abstract}

$H$ uman lungs are associated with a complex structure to perform gas exchange across a thin blood-gas interface (West, 1995). Alveolar capillary endothelial cells face toward alveolar epithelial cells on a very wide surface area via a thin basement membrane (West, 2000). Developmental disorders in this interface may result in serious respiratory problems in infants. In addition, irreversible structural changes in the blood-gas interface in mature lungs represent the most important pathophysiology of serious lung disorders such as fibrotic lung diseases and pulmonary emphysema. The recent utilization of multipotential stem cells may open the possibility of regeneration therapy (Weissman, 2000). Many premature and irreversibly destroyed lungs may be treated with this novel therapy. Therefore, understanding the normal process of human lung development is becoming a very important issue. In this process, proliferation and differentiation of the lung epithelium and airway branching have been extensively studied (Warburton

Received October 18, 2001.

Address reprint requests to: Dr. Sumiko Maeda, Department of Thoracic Surgery, Institute of Development, Aging and Cancer, Tohoku University, 4-1Seiryo-machi, Aoba-ku, Sendai, 980-8575, Japan. E-mail: sumiko@idac.tohoku.ac.jp et al, 2000), whereas the development of the pulmonary vascular system has yet to be examined in detail.

Vascularization is, in general, initiated by two different mechanisms, angiogenesis and vasculogenesis. Angiogenesis is a branching of preexisting vessels (Folkman, 1971). In contrast, vasculogenesis results from the development of endothelial lineage from putative angioblasts (Risau, 1995). Vasculogenesis is believed to be the predominant form in the very early fetal stages (Folkman, 1995), but the development of the intraparenchymal vascular system may vary among organs. For instance, vascular systems in the brain and kidney are the results of angiogenesis from the branching of central arteries (Sariola, 1983; Stone et al, 1995). The pulmonary vascular system is considered to be initiated by vasculogenesis (DeRuiter et al, 1993; Pardanaud et al, 1989; Schachtner et al, 2000) or a combination of vasculogenesis and angiogenesis (deMello, 1997; Roman, 1997). However, the temporal and spatial development of the pulmonary vascular system has yet to be fully elucidated.

The acquisition of functional phenotypes of pulmonary vessels during lung development may be very important for the functional blood-gas interface. A wide perfusion area and lower velocity of capillary blood flow allows red blood cells to contact with oxygen-rich alveolar gases for a sufficient time to 
perform gas exchange (West, 1995). Anticoagulant activity may be required to control coagulation in the pulmonary circulation. von Willebrand factor (vWF), mainly involved in primary hemostasis (Wagner, 1990), is known not to be expressed in alveolar capillary endothelial cells (Yamamoto et al, 1988). Recently, the expression of thrombomodulin (TM), an anticoagulant (Owen and Esmon, 1981), and VWF has been shown to be different between the alveolar capillary vessels and other pulmonary vessels in the adult human lung. Alveolar capillary vessels are clearly immunopositive for TM and negative for VWF, whereas other pulmonary vessels show either VWF-positive and TMnegative immunoreactivity or a mosaic pattern of these two molecules (Kawanami et al, 2000). This unique phenotype of alveolar capillary vessels may be advantageous for the blood-gas interface. However, it is not known how these phenotypes of alveolar capillary endothelial cells are determined.

One of the possible factors determining the phenotype of capillary endothelial cells is vascular endothelial growth factor (VEGF), endothelial specific proliferation, migration, and permeability factor (Ferrara and Henzel, 1989; Senger et al, 1983). VEGF is expressed in lung epithelial cells (Acarregui et al, 1999; Healy et al, 2000; Shifren et al, 1994), and recently VEGF has been demonstrated to modulate the expression of TM in cultured endothelial cells (Calnek and Grinnell, 1998).

In the present study, we characterized the structural and functional development of the vascular system in accordance with that of airways in human fetal lungs.
We examined the immunohistochemical distribution of CD34, $\alpha$-smooth muscle actin (SMA), TM, vWF, and VEGF. We used double immunohistochemistry and 3-dimensional (3-D) reconstruction techniques to demonstrate the development of the intrapulmonary vascular system in a positional interaction with branching airways.

\section{Results}

\section{Double Immunohistochemistry for CD34/SMA and 3-D Reconstruction of Developing Human Fetal Lung During the Pseudoglandular Phase}

CD34-immunopositive endothelial cells were detected in the early pseudoglandular phase lung (8 weeks gestation) (Fig. 1). Endothelial cells could be classified into two different groups by the characteristics of neighboring cells (presence or absence of SMApositive cells) and their distribution (proximal or distal lung parenchyme).

3-D-reconstructed images of double immunohistochemistry for CD34/SMA demonstrated that two types of endothelial cells distribute differently in the fetal lung. Endothelial cells (green) associated with SMApositive cells (red) formed a tube-like structure and were located next to the airways (blue) in the early pseudoglandular phase lung (8 weeks gestation). In contrast, endothelial cells without SMA-positive cells were distributed diffusely throughout the lung mesenchyme. The anastomosis between the two different types of endothelial cells (presence or absence of

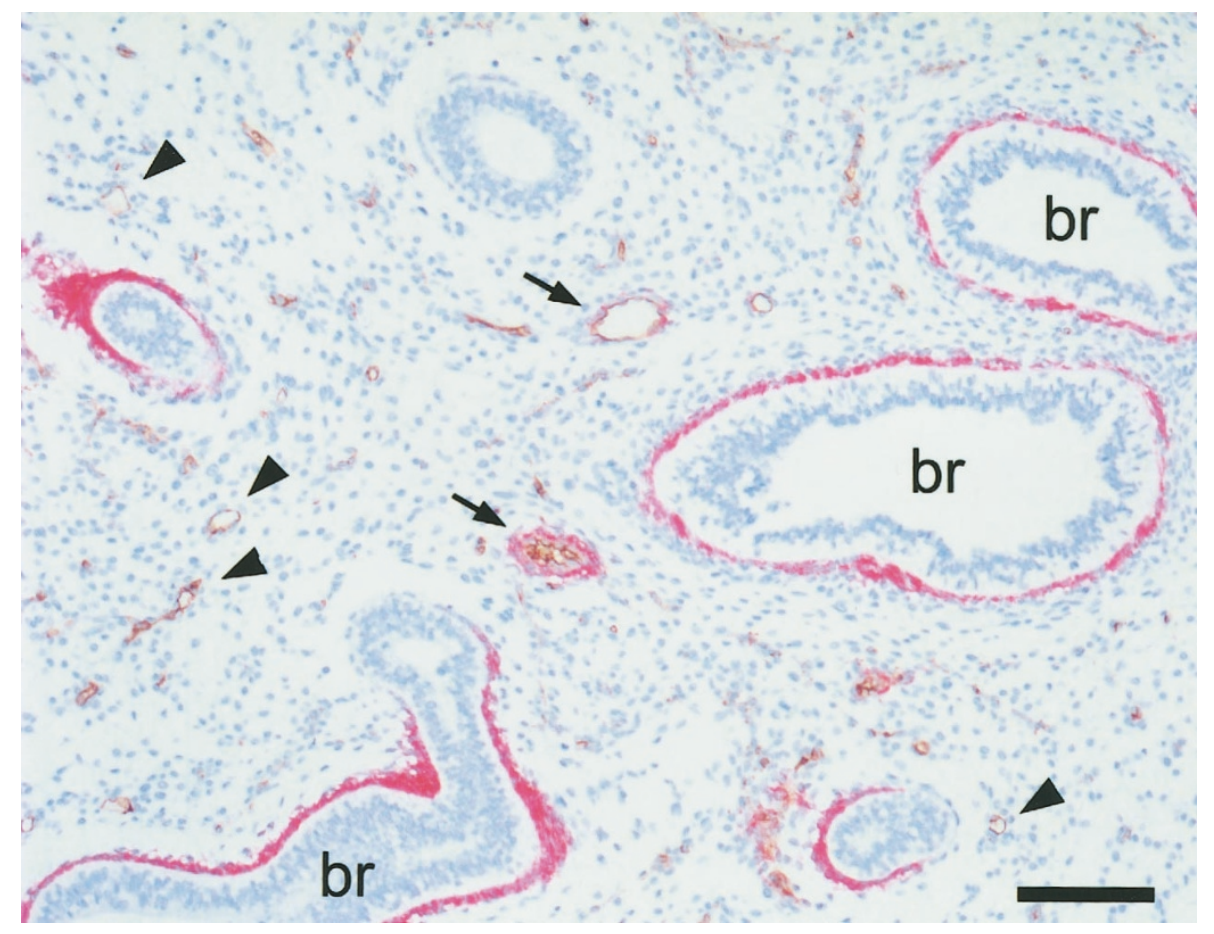

Figure 1.

Double immunohistochemistry for CD34 and $\alpha$-smooth muscle actin (SMA) in a human fetal lung specimen obtained from the early pseudoglandular phase (8 weeks gestation). Immunoreactivity for CD34 and SMA are visualized as brown and red, respectively. Gland-like structures are fetal bronchi (br). Two different types of endothelial cells are noted in this picture. One is associated with SMA-positive cells (arrows) and the other is without SMA-positive cells (arrowheads). Bar indicates $50 \mu \mathrm{m}$. 


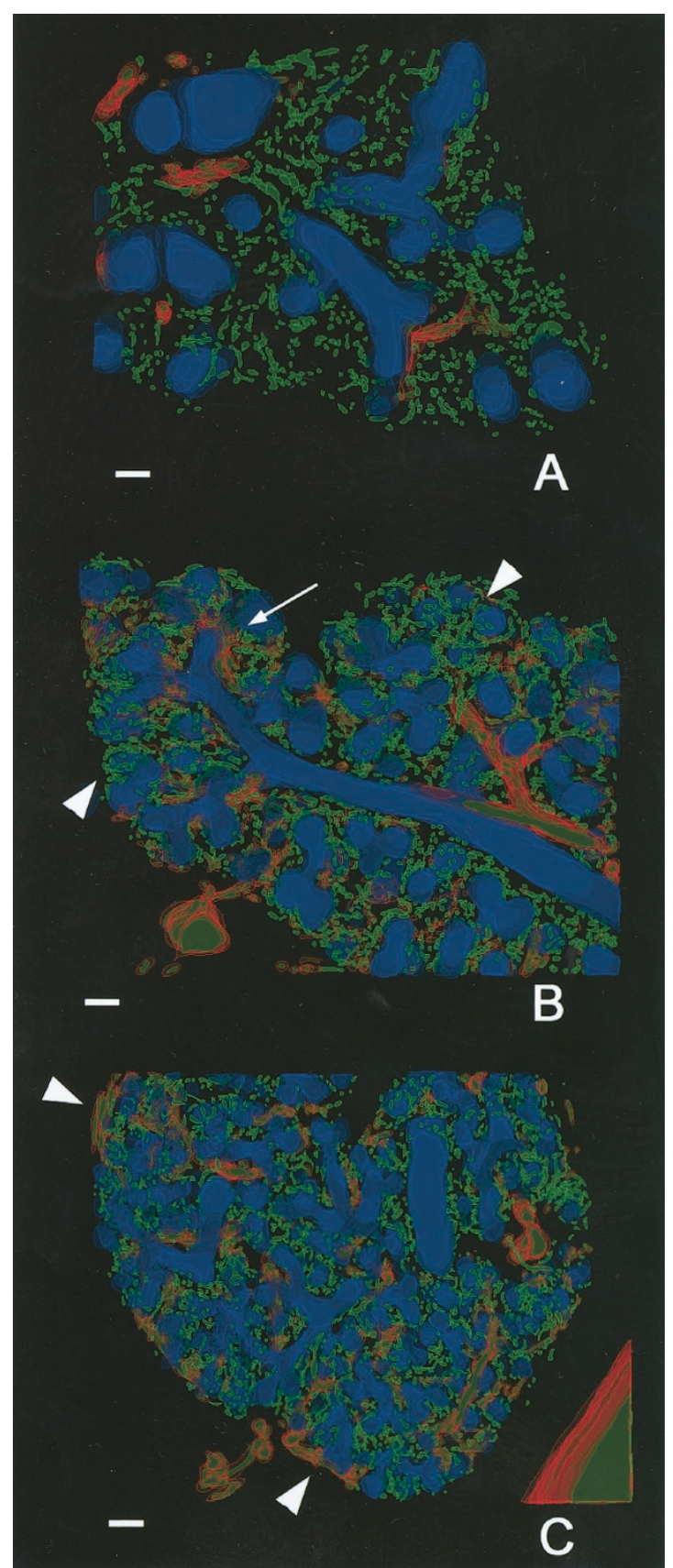

Figure 2.

Three-dimensional (3-D) reconstruction of double-immunostained lung sections. CD34 and SMA are visualized as green and red, respectively. The developing bronchi are shown in blue. $A$, In the early pseudoglandular phase lung (8 weeks gestation), CD34-positive endothelial cells distribute diffusely in the lung mesenchyme, whereas vessels associated with SMA immunoreactivity are only seen in the neighboring bronchi. Communication between the two different types of endothelial cells (presence or absence of SMA-positive cells) is not apparent. B, In the mid-pseudoglandular phase lung (13 weeks gestation), fetal bronchi increases the number of branches, and endothelial cells without SMA-positive cells seem to form network structures around the budding components of bronchi (arrowheads). Large pulmonary vessels (associated with SMA-positive cells) extend their length along the bronchi toward peripheral budding components and then connect to the network structure of endothelial cells (arrow). C, In the late pseudoglandular phase lung (16 weeks gestation), new large vessels (associated with SMA-positive cells) are present under the subpleural area (arrowheads). Note the increased blue area and the close contact with the network structure of endothelial cells and bronchi. Total thickness of the reconstructed images is $50 \mu \mathrm{m}$. Bar indicates $100 \mu \mathrm{m}$.
SMA-positive cells) was not apparent (Fig. 2A). In the mid-pseudoglandular phase lung (13 weeks gestation), fetal bronchi was demonstrated to increase the number of branches, and endothelial cells without SMA-positive cells formed network structures around the budding components of bronchi. Large pulmonary vessels (associated with SMA-positive cells) extended along bronchi toward the distal lung parenchyme, and several of these anastomosed with the network structure of endothelial cells (Fig. 2B). In the late pseudoglandular phase lung (16 weeks gestation), new large vessels, associated with SMA-positive cells, appeared under the subpleural area. The blue area expanded, and the network structure of endothelial cells and bronchi came in close proximity (Fig. 2C).

\section{Distribution of TM and vWF in the Fetal Pulmonary Vessels}

In the pseudoglandular phase lung, only a few CD34positive endothelial cells expressed TM (Fig. 3, A and B). In contrast, vWF was positive in almost all CD34positive endothelial cells (Fig. 3C). In the canalicular phase lung, in addition to the increase in the number of CD34-positive cells in the distal area (Fig. 3D), there was also an increase in TM immunoreactivity (Fig. 3E). VWF immunoreactivity was distributed in both types of vessels; one was a muscular vessel-like appearance associated with SMA-positive cells and the other was a capillary-like network not surrounded by SMApositive cells (Fig. 3F). In the terminal sac phase lung, primitive alveolar capillary vessels were detected (Fig. $3 G)$. Primitive alveolar capillary endothelial cells were immunopositive for TM (Fig. 3H) but negative for vWF (Fig. 3I). This immunohistochemical distribution of TM and $\mathrm{VWF}$ in the terminal sac phase lung was very similar to that of mature lungs (Fig. 3, J-L; 61-year-old male).

Morphometric analysis indicated that approximately $70 \%$ of total lung vessels existed as capillary-like structures in the pseudoglandular phase lung, and this ratio increased to almost $90 \%$ in the terminal sac phase lung (pseudoglandular phase, $0.708 \pm 0.028$ [mean \pm SEM]; canalicular phase, $0.750 \pm 0.025$; terminal sac phase, $0.868 \pm 0.002$ ) (Fig. 4). The ratio of TM-positive capillary vessels also increased from $54 \%$ to $80 \%$ (pseudoglandular phase, $0.542 \pm 0.017$; canalicular phase, $0.562 \pm 0.022$; terminal sac phase, $0.795 \pm 0.009$ ). In contrast, the ratio of vWF-positive capillaries gradually decreased from $60 \%$ to $28 \%$ throughout fetal lung development (pseudoglandular phase, $0.595 \pm 0.010$; canalicular phase, $0.448 \pm$ 0.044; terminal sac phase, $0.275 \pm 0.027$ ) (Fig. 4).

\section{Immunohistochemistry for VEGF in the Developing Human Lung}

VEGF immunoreactivity was detected in the cytoplasm of airway epithelial cells throughout the branching bronchi, and more intense expression was observed in the budding component of airways in the canalicular phase lung (Fig. 5). 

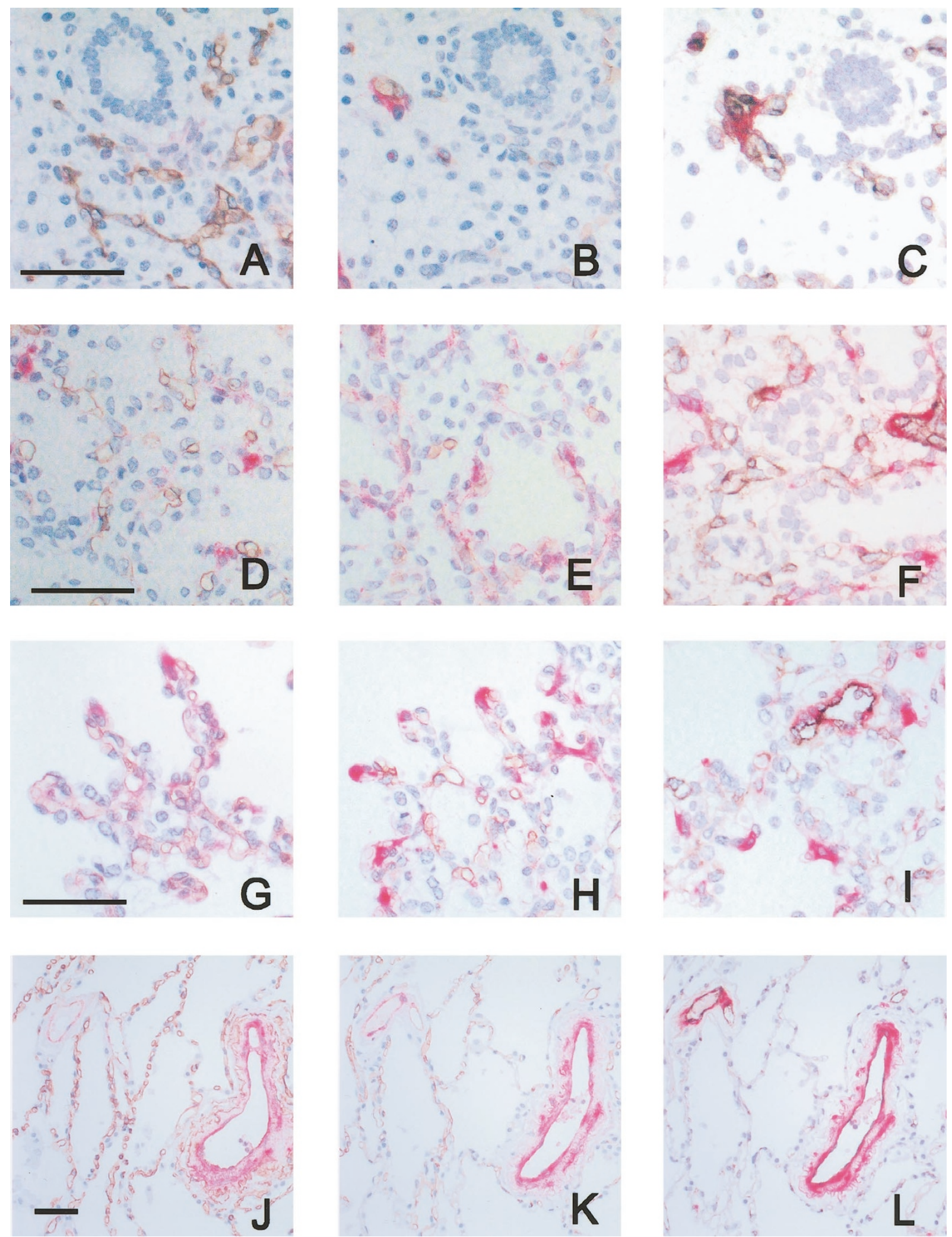

Figure 3.

Change of immunohistochemical distribution for thrombomodulin (TM) and von Willebrand factor (vWF) in the fetal lung. Pictures in the left column (A, D, G, and J) show double immunohistochemistry for CD34 (brown) and SMA (red) in the pseudoglandular phase lung (15 weeks gestation), the canalicular phase lung (21 weeks gestation), the terminal sac phase lung (38 weeks gestation), and in an adult lung (61-year-old, male), respectively. Pictures in the middle column (B, E, $\mathrm{H}$, and $\mathrm{K}$ ) and in the right column (C, F, I, and L) show the immunoreactivity for TM (brown) and vWF (brown) with SMA staining (red) at different developmental stages. The number of TM-positive cells progressively increases in capillary networks of CD34-positive endothelial cells. In contrast, vWF immunoreactivity is localized in large vessels associated with SMA-positive cells at the terminal sac phase, although endothelial cells without SMA-positive cells express vWF at earlier developmental stages. 


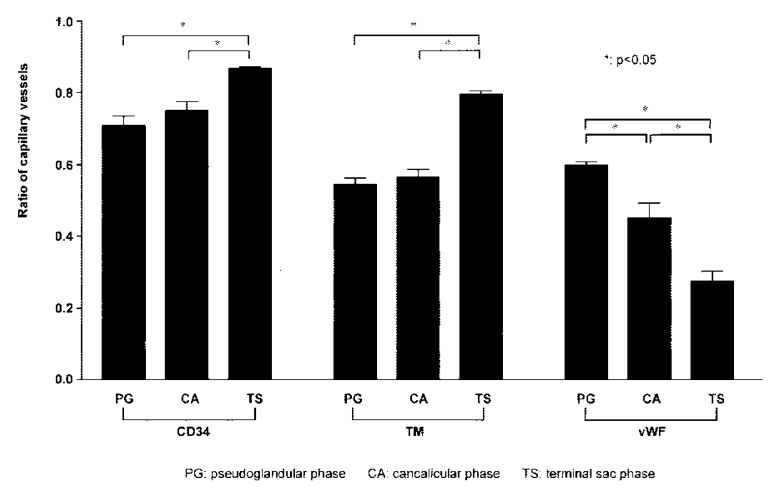

Figure 4.

Ratio of SMA-negative vessels in each double-immunostained section. Approximately $70 \%$ of total lung vessels exist as capillary vessels (not surrounded by SMA-positive cells) during the pseudoglandular and canalicular phases, and this ratio was further increased to almost $90 \%$ at the terminal sac phase. The ratio of TM-positive capillary vessels was shown to markedly increase, whereas they lose VWF immunoreactivity in the terminal sac phase. Data are presented as mean \pm SEM for $n=4$ in each stage of development. * $p$ $<0.05$

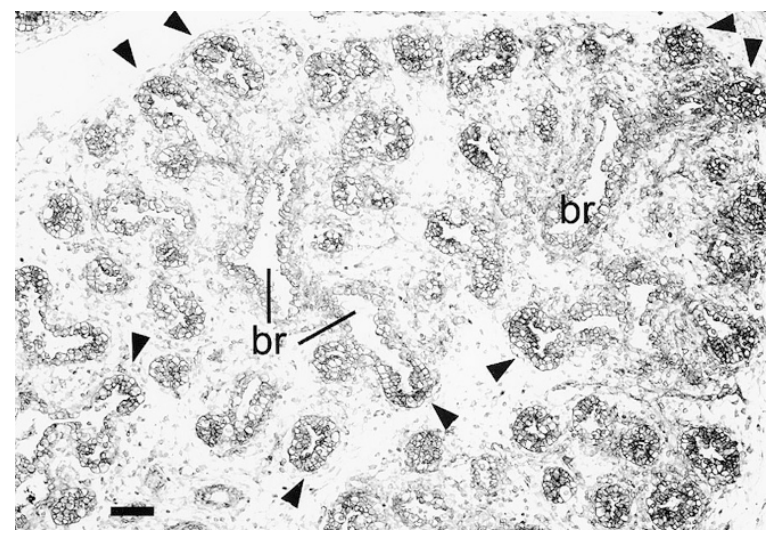

Figure 5.

Immunohistochemistry for vascular endothelial growth factor (VEGF) in the canalicular phase lung. VEGF is expressed in the epithelial cells throughout the branching bronchi $(b r)$, and more intense expression is seen at the budding components of distal airways (arrowheads).

\section{Discussion}

\section{Structural Development of Intrapulmonary Circulation}

It is well known that the human pulmonary arterial system is composed of three different origins, ie, pulmonary trunk derived from the septation of the truncus arteriosus, right and left main pulmonary arteries from the sixth paired aortic arch, and the intrapulmonary arteries from primitive vessels associated with budding airways (Cucci et al, 1964). However, it remains unclear how intrapulmonary arteries finally establish pulmonary circulation (DeRuiter et al, 1993; Pardanaud et al, 1989; Sariola et al, 1983; Schachtner et al, 2000; Stone et al, 1995). Results of our present study suggest that intrapulmonary arteries contact with the preexisting capillary network, which develops independently from large intrapulmonary vessels. By utilizing double immunohistochemistry for CD34 and SMA, we demonstrated that endothelial cells at an early pseudoglandular phase (8 weeks gestation) can be classified into two different groups by the presence or absence of SMA-positive cells as their neighboring cells. 3-D reconstruction of the double-immunostained lung sections further demonstrated that endothelial cells surrounded by SMApositive cells formed proximal large vessels by "angiogenesis" during the mid-pseudoglandular phase (13 weeks gestation). In contrast, endothelial cells without SMA-positive cells in the distal area seemed to develop the capillary network by "vasculogenesis." Finally, these differently developed endothelial cells seemed to be connected in the late pseudoglandular phase (16 weeks gestation).

Previous anatomical studies utilizing the pulmonary vascular cast and angiogram in developing mouse lungs demonstrated that development of intrapulmonary arteries follows the steps of extension, branching, and anastomosis to the capillary network (deMello et al, 1997). However, vascular casts and angiograms provide no information as to the alveolar capillary network during development, when it is separated from the pulmonary arteries. Therefore, we used a $3-D$ reconstruction technique in this study. This approach may be time-consuming and labor intensive, but it can provide more pivotal information in understanding the vascular structure of the developing human lung. In addition, immunohistochemistry and its 3-dimensionally reconstructed images can provide the functional information and the exact distribution of the cells. This technique has enabled the characterization of the plexiform lesion of pulmonary arteries in primary pulmonary hypertension patients (Cool et al, 1999).

Results of 3-D reconstruction in our study clearly demonstrated that endothelial cells themselves formed capillary networks in the distal lung parenchyme before the anastomosis with intrapulmonary arteries. We could not determine the origin of proximal vessels, whether an ingrowth of the main pulmonary artery or a possible transformation of the lung mesenchymal cells. However, our results straightly suggest that endothelial cells in the distal lung parenchyme, separated from proximal vessels, may be derived from lung mesenchymal cells, which are demonstrated to transform into endothelial cells (Akeson et al, 2000).

\section{Alteration of Endothelial Phenotypes in Pulmonary Vessels}

CD34-positive endothelial cells as well as lung epithelial cells progressively increased in number throughout lung development. Approximately $70 \%$ of total lung endothelial cells exist as capillary vessels (not surrounded by SMA-positive cells) during the pseudoglandular and canalicular phases. This ratio was further increased to almost $90 \%$, when the capillary network was closely located in conjunction with the budding component of distal airways in the terminal sac phase. This alveolus-like structure may not be sufficient for normal respiration, but premature infants delivered during the terminal sac phase can be successfully treated with intensive respiratory care. This 
fact suggests that the pulmonary circulation has been established with a functional blood-gas interface as well as sufficiently wide surface area by the latest gestational stage. Results of our study also demonstrated that the number of endothelial cells positive for TM markedly increased in capillary vessels around the budding components of distal airways, whereas immunoreactivity for VWF decreased during the terminal sac phase. These findings all suggest that the unique immunohistochemical characteristic of alveolar capillary endothelial cells in the mature lung is developmentally programmed.

TM is a surface protein that binds with thrombin and activates protein $\mathrm{C}$ and functions as an anticoagulant (Owen and Esmon, 1981). TM may play an important role in controlling coagulation in the pulmonary circulation system after birth. TM expression is altered in some pathologic conditions. For instance, alveolar capillary vessels have been reported to express very low levels of TM in inflammatory lung conditions such as diffuse alveolar damages (Matsubara et al, 1995). Several studies on cultured endothelial cells have demonstrated that the expression of TM is diminished by inflammatory cytokines (Fujiwara et al, 2001; Lentz et al, 1991). Several in vitro experiments suggest that shear stress may change the characteristics of endothelial cells (Golledge et al, 1999; Malek et al, 1994). Recent gene analysis of patients with primary pulmonary hypertension using microarray also revealed the down-regulation of TM gene in the lung (Geraci et al, 2001). We recently examined the histology of a resected lung in a case of unilateral absence of pulmonary artery, where alveolar capillary vessels were exposed to high blood pressure via bronchial arteries. Lung specimens in this unilateral absence of pulmonary artery case expressed less TM, whereas vWF was positive (Maeda et al, 2001). Increased pressure and shear stress alone, however, cannot account for the alterations of immunohistochemical characteristics of endothelial cells during normal lung development, because the pulmonary circulation in terminal sac phase remains a high-pressure circulatory system.

We also found in this study that endothelial cells of large vessels, which were in close proximity to airways, maintained VWF immunoreactivity throughout all developmental stages. Capillaries in the distal lung area were also immunopositive for VWF at the early pseudoglandular phase, although they changed to a TM-predominant phenotype. These findings suggest that endothelial cells may first express vWF in the developing human lung. Expression of vWF has been reported to be dependent on the environment where endothelial cells exist (Aird et al, 1997). This vascular bed-specific regulation of vWF may be involved in normal lung development.

\section{VEGF Expression in Fetal Lung Epithelial Cells}

In the present study, we demonstrated that VEGF was expressed in the epithelial cells throughout the branching airways, with more intense immunoreactiv- ity detected in the budding components of distal airways. We also demonstrated that endothelial cells of the capillary network surrounding the distal airway developmentally altered their characteristics to be TM-predominant types. A recent study on cultured endothelial cells demonstrated that the expression of TM and TM-dependent anticoagulant activity is regulated by VEGF (Calnek and Grinnell, 1998). These findings suggest that capillary endothelial cells around the budding components of distal airways may therefore be exposed to relatively high concentrations of VEGF, resulting in the acquisition of TM-predominant phenotypes in these endothelial cells. In addition, VEGF has been demonstrated to play a role in maintaining the alveolar structure (Kasahara et al, 2000). All these findings suggest that diffusely distributed VEGF in pulmonary epithelial cells is involved in the endothelial proliferation and the maintenance of vascular structure, and relatively abundant VEGF in the budding components of distal airway may change the endothelial cell phenotype to the mature alveolar capillary vessel type.

Our results in this study demonstrated that the most distal epithelial cells in the developing human lung regulate the functional phenotype of endothelial cells in the capillary network via VEGF. This process may be very important in establishing the sufficiently wide and functional blood-gas interface. A better understanding of the process of normal lung development can therefore provide important clues for a novel therapy to treat many patients with premature and irreversibly destroyed lungs.

\section{Materials and Methods}

\section{Lung Tissues}

Human fetal lung tissues $(n=12)$ were obtained from elective abortions at 8 to 21 weeks gestation of normal pregnancy. Five specimens from 22 weeks of gestation to term $(23,31,33,35$, and 38 weeks gestation) were retrieved from autopsy files at Tohoku University Hospital. The fetal age was estimated by the last menstrual date, body weight, and/or crown-rump length. Human adult lung tissues $(n=6)$ were obtained from surgical specimens. For immunohistochemical analysis, lung tissues were fixed in 10\% formalin, embedded in paraffin, and sectioned at 2.5- $\mu \mathrm{m}$ thickness. This study protocol was approved by the Committee on Ethics of Tohoku University School of Medicine, Sendai, Japan.

\section{Antibodies}

The antibodies used in this study were as follows: monoclonal antibody for CD34 (clone 4A1; Nichirei Company Ltd., Tokyo, Japan); monoclonal antibodies for TM (TM1009) and SMA (clone 1A4), polyclonal antibody for vWF (lot105; DAKO Company Ltd., Glostrup, Denmark); and monoclonal antibody for VEGF (C-1; Santa Cruz Biotechnology, Inc., California). 


\section{Immunohistochemistry}

Immunohistochemical analysis was performed using the streptavidin-biotin amplified method with a Histofine Kit (Nichirei). The optimal dilutions of primary antibodies were as follows: CD34, 1/100; TM, 1/100; VWF, 1/2000; SMA, 1/1000; and VEGF, 1/500. Deparaffinized sections were incubated with $0.3 \%$ hydrogen peroxide in absolute methanol for 30 minutes to block endogenous peroxidase activity. For antigen retrieval for $\mathrm{VWF}$, deparaffinized sections were treated with trypsin $\left(1 \mathrm{mg} / \mathrm{ml}\right.$ trypsin, $1 \mathrm{mg} / \mathrm{ml} \mathrm{CaCl}_{2}$ in $0.05 \mathrm{M}$ Tris- $\mathrm{HCl}$ buffer, $\mathrm{pH}$ 7.6). The antigen-antibody complex was visualized with 3.3'-diaminobenzidinetetrachloride (DAB) solution (1 mM DAB, 0.05 M Tris$\mathrm{HCl}$ buffer $\mathrm{pH} 7.6$, and $\left.0.006 \% \quad \mathrm{H}_{2} \mathrm{O}_{2}\right)$ and counterstained with methyl green. Tissue sections of tonsil were used as positive controls for CD34 and vWF, those of normal adult lung for TM and SMA, and that of breast cancer for VEGF. Tissue sections were incubated with normal mouse or rabbit immunoglobulin $G$ instead of primary antibodies for negative control of immunohistochemistry. No specific immunoreactivity was detected in these tissue sections.

\section{Double Immunostaining}

Double immunostaining for CD34/SMA and TM/SMA was performed as described previously (Suzuki, 1998) with slight modifications. In brief, deparaffinized sections were treated with $1 \%$ normal goat serum for 20 minutes at room temperature and incubated with anti-CD34 or TM antibody at $4^{\circ} \mathrm{C}$ for 18 hours. After blocking endogenous peroxidase activity, the slides were subsequently reacted with EnVision labeled with peroxidase (DAKO) for 90 minutes at room temperature. The immune complex was visualized with $D A B$ solution. The sections were then placed in boiling 0.01 $\mathrm{M}$ citric acid buffer $(\mathrm{pH}$ 6.0) for 5 minutes and were treated with $1 \%$ normal goat serum for 20 minutes. Reacted sections were subsequently incubated with the antibody for SMA at $4^{\circ} \mathrm{C}$ for 18 hours. The slides were reacted with EnVision labeled with alkaline phosphatase (DAKO) and visualized by Vector Red (Vector Laboratories, Burlingame, California) in $0.1 \mathrm{M} \mathrm{Tris-HCl}$ buffer ( $\mathrm{pH}$ 8.2). Hematoxylin was used for counterstaining. For double immunostaining of vWF/SMA, immunoreactivity for SMA was visualized with Vector Red in the first step. Reacted tissue sections were then boiled in citrate buffer and subsequently treated with trypsin. Sections were then treated with $1 \%$ normal goat serum for 20 minutes at room temperature and incubated with anti-vWF antibody at $4^{\circ} \mathrm{C}$ for 18 hours. After the blocking of endogenous peroxidase activity, sections were reacted with EnVision labeled with peroxidase and the immune complex was visualized with DAB solution.

For negative controls, sections were incubated with 0.01 M PBS or normal mouse or rabbit IgG instead of primary antibodies. Both of these control procedures consistently yielded negative results. Immunoreactivity was compared with the immunohistochemistry for each single antigen to confirm the result of double immunostaining.

\section{3-D Reconstruction of the Fetal Lung}

3-D reconstruction of blood vessels and airways of fetal lungs during the pseudoglandular phase was performed from serial tissue sections of double immunostaining for CD34/SMA with the aid of a computer system (Mate NX, MA3OH, NEC). The first of the serial sections was projected onto a sheet of tracing paper at a magnification of $\times 200$ using a profile projector (Nikon, V-16C). The contours of the blood vessels, airway epithelial basement membrane, and SMA positive area were faithfully delineated. This procedure was repeated for the next section until all the serial sections were reduced to a series of line drawings. These drawings were then captured into a computer by tracing their contours on a digitizer (DIGITIZER II, UD-1218-RE; Wacom Company Ltd., Saitama, Japan). 3-D images were generated by utilizing special software (OZ95; Rise Company Ltd., Sendai, Japan). The coordination of serial pictures was performed by the "best fit" methods, selecting the most adequate position by inspection with regard to its previous image (Yaegashi, 1987).

\section{Morphometrical Analysis}

Evaluation of immunopositive vessels was identically performed by two of the authors (S. M. and T. S) after selecting 10 high-power fields simultaneously using double-headed light microscopy. Double immunostaining for CD34/SMA was evaluated by counting the number of total CD34-immunopositive vessels and SMA-negative vessels in CD34-positive cells. We then calculated the value of (SMA negative vessels in CD34-positive cells)/(total CD34-positive vessels). This value represents the relative ratio of capillary vessels to the total number of vessels in fetal lung. The same procedure was applied to doubleimmunostained sections for TM/SMA and VWF/SMA. Interobserver differences were less than $5 \%$, and the mean of the two values was obtained.

\section{Statistical Analysis}

Data of morphometric analysis are presented as a mean \pm SEM. Statistical analysis was performed using the Stat-View J-4.5 software package (Abacus Concepts, Inc., Berkeley, California). The values obtained in the different groups were compared using one-way ANOVA and post-hoc test (Scheffé test). Significance was set at $p<0.05$.

\section{References}

Acarregui MJ, Penisten ST, Goss KL, Ramirez K, and Snyder JM (1999). Vascular endothelial growth factor gene expression in human fetal lung in vitro. Am J Respir Cell Mol Biol $20: 14-23$. 
Aird WC, Edelberg JM, Weiler-Guettler H, Simmons WW, Smith TW, and Rosenberg RD (1997). Vascular bed-specific expression of an endothelial cell gene is programmed by the tissue microenvironment. J Cell Biol 138:1117-1124.

Akeson AL, Wetzel B, Thompson FY, Brooks SK, Paradis $\mathrm{H}$, Gendron RL, and Greenberg JM (2000). Embryonic vasculogenesis by endothelial precursor cells derived from lung mesenchyme. Dev Dyn 217:11-23.

Calnek DS and Grinnell BW (1998). Thrombomodulindependent anticoagulant activity is regulated by vascular endothelial growth factor. Exp Cell Res 238:294-298.

Cool CD, Stewart JS, Werahera P, Miller GJ, Williams RL, Voelkel NF, and Tuder RM (1999). Three-dimensional reconstruction of pulmonary arteries in plexiform pulmonary hypertension using cell-specific markers: Evidence for a dynamic and heterogeneous process of pulmonary endothelial cell growth. Am J Pathol 155:411-419.

Cucci CF, Doyle EF, and Lewis EW Jr (1964). Absence of primary division of the pulmonary trunk: An ontogenetic theory. Circulation 29:124-131.

deMello DE, Sawyer D, Galvin N, and Reid LM (1997). Early fetal development of lung vasculature. Am J Respir Cell Mol Biol 16:568-581.

DeRuiter MC, Poelmann RE, Mentink MM, Vaniperen L, and Gittenberger-De Groot AC (1993). Early formation of the vascular system in quail embryos. Anat Rec 235:261-274.

Ferrara N and Henzel WJ (1989). Pituitary follicular cells secrete a novel heparin-binding growth factor specific for vascular endothelial cells. Biochem Biophys Res Commun 161:851-858.

Folkman J (1971). Tumor angiogenesis: Therapeutic implications. N Engl J Med 285:1182-1186.

Folkman J (1995). Angiogenesis in cancer, vascular, rheumatoid and other disease. Nat Med 1:27-31.

Fujiwara M, Jin E, Ghazizadeh M, and Kawanami O (2001). An in vitro model to evaluate regulatory mechanisms of antigen expression by normal pulmonary vessel endothelial cells. Microvasc Res 61:215-219.

Geraci MW, Moore M, Gesell T, Yeager ME, Alger L, Golpon H, Gao B, Loyd JE, Tuder RM, and Voelkel NF (2001). Gene expression patterns in the lungs of patients with primary pulmonary hypertension: a gene microarray analysis. Circ Res 88:555-562.

Golledge J, Turner RJ, Gosling M, and Powell JT (1999). Rapid changes in the coagulant proteins on saphenous vein endothelium in response to arterial flow. Angiology 50:693701.

Healy AM, Morgenthau L, Zhu X, Farber HW, and Cardoso WV (2000). VEGF is deposited in the subepithelial matrix at the leading edge of branching airways and stimulates neovascularization in the murine embryonic lung. Dev Dyn 219: 341-352.

Kasahara Y, Tuder RM, Taraseviciene-Stewart L, Le Cras TD, Abman S, Hirth PK, Waltenberger J, and Voelkel NF (2000). Inhibition of VEGF receptors causes lung cell apoptosis and emphysema. J Clin Invest 106:1311-1319.

Kawanami O, Jin E, Ghazizadeh M, Fujiwara M, Jiang L, Ohaki Y, Gomibuchi M, and Takemura T (2000). Mosaic-like distribution of endothelial cell antigens in capillaries and juxta-alveolar microvessels in the normal human lung. Pathol Int 50:136-141.

Lentz SR, Tsiang M, and Sadler JE (1991). Regulation of thrombomodulin by tumor necrosis factor-alpha: Comparison of transcriptional and posttranscriptional mechanisms. Blood 77:542-550.

Maeda S, Suzuki S, Moriya T, Suzuki T, Chida M, Suda H, Sakuma H, Kondo T, and Sasano H (2001). Isolated unilateral absence of a pulmonary artery: Influence of systemic circulation on alveolar capillary vessels. Pathol Int 51:649-653.

Malek AM, Jackman R, Rosenberg RD, and Izumo S (1994). Endothelial expression of thrombomodulin is reversibly regulated by fluid shear stress. Circ Res 74:852-860.

Matsubara O, Tamura A, Ohdama S, and Mark EJ (1995). Alveolar basement membrane breaks down in diffuse alveolar damage: An immunohistochemical study. Pathol Int 45: 473-482.

Owen WG and Esmon CT (1981). Functional properties of an endothelial cell cofactor for thrombin-catalyzed activation of protein C. J Biol Chem 256:5532-5535.

Pardanaud L, Yassine F, and Dieterlen-Lievre F (1989). Relationship between vasculogenesis, angiogenesis and haemopoiesis during avian ontogeny. Development 105:473485.

Risau W (1995). Differentiation of endothelium. FASEB J 9:926-933.

Roman J (1997). Cell-cell and cell-matrix interactions in development of the lung vasculature. In: MacDonald JA, editors. Lung growth and development. New York: Marcel Dekker, 365-399.

Sariola H, Ekblom P, Lehtonen E, and Saxen L (1983). Differentiation and vascularization of the metanephric kidney grafted on the chorioallantoic membrane. Dev Biol 96:427435.

Schachtner SK, Wang Y, and Scott Baldwin H (2000). Qualitative and quantitative analysis of embryonic pulmonary vessel formation. Am J Respir Cell Mol Biol 22:157-165.

Senger DR, Galli SJ, Dvorak AM, Perruzzi CA, Harvey VS, and Dvorak HF (1983). Tumor cells secrete a vascular permeability factor that promotes accumulation of ascites fluid. Science 219:983-985.

Shifren JL, Doldi N, Ferrara N, Mesiano S, and Jaffe RB (1994). In the human fetus, vascular endothelial growth factor is expressed in epithelial cells and myocytes, but not vascular endothelium: Implications for mode of action. J Clin Endocrinol Metab 79:316-322.

Stone J, Itin A, Alon T, Pe'er J, Gnessin H, Chan-Ling T, and Keshet $E$ (1995). Development of retinal vasculature is mediated by hypoxia-induced vascular endothelial growth factor (VEGF) expression by neuroglia. J Neurosci 15:4738-4747.

Suzuki T, Sasano H, Takaya R, Fukaya T, Yajima A, and Nagura $H$ (1998). Cyclic changes of vasculature and vascular phenotypes in normal human ovaries. Hum Reprod 13:953959.

Wagner DD (1990). Cell biology of von Willebrand factor. Annu Rev Cell Biol 6:217-246.

Warburton D, Schwarz M, Tefft D, Flores-Delgado G, Anderson KD, and Cardoso WV (2000). The molecular basis of lung morphogenesis. Mech Dev 92:55-81. 
Weissman IL (2000). Translating stem and progenitor cell biology to the clinic: Barriers and opportunities. Science 287:1442-1446.

West JB (1995). Structure and function: How the architecture of the lung subserves its function. In: Satterfield TS, editor. Respiratory physiology: The essentials, 5th ed. Baltimore: Williams \& Wilkins, 1-10.

West JB (2000). Invited review: Pulmonary capillary stress failure. J Appl Physiol 89:2483.
Yaegashi H, Takahashi T, and Kawasaki M (1987). Microcomputer-aided reconstruction: A system designed for the study of 3-D microstructure in histology and histopathology. J Microsc 146:55-65.

Yamamoto M, Shimokata K, and Nagura H (1988). An immunohistochemical study on phenotypic heterogeneity of human pulmonary vascular endothelial cells. Virchows Arch A Pathol Anat Histopathol 412:479-486. 